\title{
Transitional cell carcinoma developing in a bladder diverticulum: Early diagnosis by computed tomography
}

\author{
J Shah \\ Department of Radiology, GT Hospital, Mumbai, India \\ J Shah, MD
}

Corresponding author: J Shah (shahjeshil@yahoo.com)

Computed tomography confirmed a left-sided narrow neck urinary bladder diverticulum, with wall thickening, in a 56-year-old man. These findings were initially detected on ultrasonographic investigation. Transitional cell carcinoma was confirmed histologically. There is an increased incidence of neoplastic transformation in urinary bladder diverticuli, and therefore an early search for subtle imaging signs should be implemented.

S Afr J Rad 2013;17(1):39-40. DOI:10.7196/SAJR.788

\begin{abstract}
A 56-year-old man presented with painless haematuria and occasional right-sided flank pain. Routine investigations were non-contributory, with serum creatinine levels of $1.4 \mathrm{mg} / \mathrm{dl}$. Sonography showed left-sided grade 1 hydronephrosis and a dilated left ureter. A narrow neck urinary bladder diverticulum was identified near the left vesico-ureteric junction. The left terminal ureter tapered abruptly, medial to the bladder diverticulum.

The patient was referred for a CT scan, which confirmed the narrow neck bladder diverticulum and identified minimal thickening of the
\end{abstract}

medial wall, resulting in left ureteric dilatation with hydronephrosis. There was minimal enhancement of the medial diverticular wall, which was initially not appreciated on the 5-minute delayed phase scan, owing to the layering effect of excreted contrast in the bladder lumen. However, it was identified on the prone delayed-phase CT examination (Fig. 1D). The finding on this image prompted cystoscopy and guided biopsy. Subsequent histopathological examination confirmed a transitional cell carcinoma (TCC). The diverticulum was surgically removed, with wide 


\section{RADIOLOGICAL TIPS}

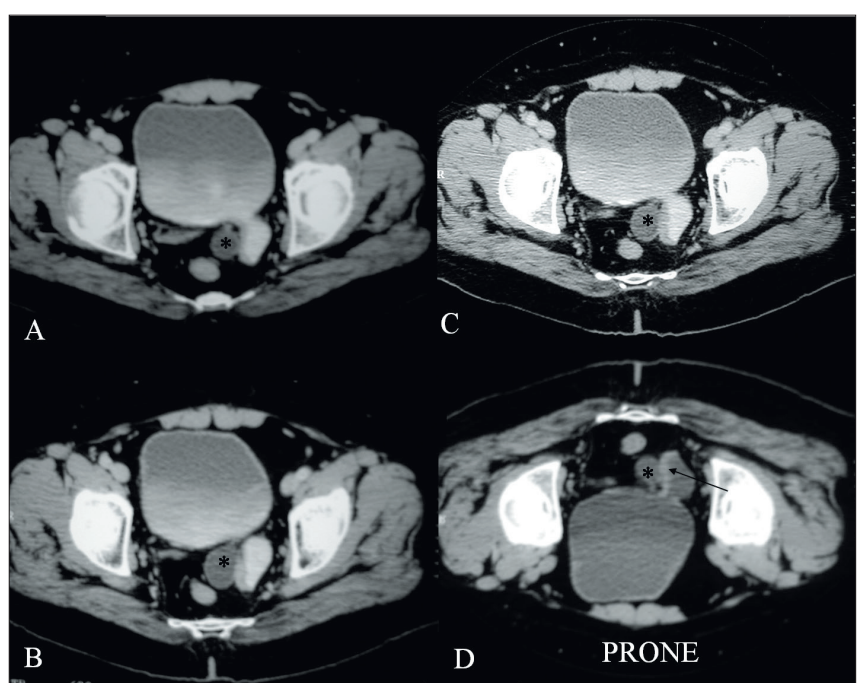

Fig. 1. Axial post-contrast CT scans obtained in $(a-c)$ supine and $(d)$ prone positions show minimal thickening and enhancement of the medial wall of a left-sided narrow neck bladder diverticulum. The asterisk indicates the dilated lower left ureter.

margins, and the left ureter was re-anastomosed onto the bladder. The histopathology report suggested a stage Ta tumor. The postoperative stay was uneventful, and the patient was discharged on the 7 th day post surgery.

\section{Discussion}

Neoplasms arising in urinary bladder diverticulae are uncommon, but not rare. Of patients with vesical diverticulae, 2 - 7\% show neoplasm development within the diverticulae ${ }^{[1]}$ Although cystoscopy is a reliable method for diagnosing most bladder neoplasms, certain diverticulae are inaccessible or may be missed, particularly those with a narrow orifice. Imaging is therefore an important diagnostic tool. ${ }^{[1]}$ Bladder diverticulum neoplasms are characterised by early transmural invasion and a tendency for higher histopathological grades, which make prompt diagnosis and treatment crucial. Filling defects caused by these tumours are not always visualised on intravenous urography and/or cystography. Cross-sectional imaging methods, including ultrasonography, $\mathrm{CT}$ and magnetic resonance imaging (MRI) have to be used singly or in combination, in neoplasms of the lower urinary tract. ${ }^{[1,2]}$ Urinary stasis with chronic infection and inflammation leads to the development of dysplasia, leukoplakia and squamous cell metaplasia in approximately $80 \%$ of all diverticulae. ${ }^{[2]}$ These histological abnormalities may precede the development of vesical diverticular neoplasia. The most common histological tumour type is TCC (about 78\%), followed by squamous cell carcinoma (SCC) (17\%), a combination of TCC and SCC types (2\%) and adenocarcinoma (2\%). Rarer forms such as metastases, sarcoma, etc. have also been described. ${ }^{[1,2,4]}$

Bladder tumours are more common in men, the incidence increasing with age. ${ }^{[5]}$ Local endoscopic transurethral resection and intravesical instillation of adjuvant chemotherapeutic agents/Bacillus Calmette-Guérin, an immune-modulating agent, are employed for superficial lesions. More invasive tumours are treated with cystectomy, chemotherapy and/or radiation. Transurethral resection can be complicated by narrow ostia and thin diverticular walls. Diverticulectomy, partial cystectomy and radical cystectomy with or without intravesical instillations are other modes of treatment. ${ }^{[5]}$

In view of an increased incidence of malignant transformation within bladder diverticulae, periodic screening should be considered in such patients. Further research is required to determine which imaging modality (ultrasound or CT) would be the most appropriate. Appropriate screening intervals must be determined. Any suspicious early thickening or enhancement of the walls, as seen in this case, should be followed with cystoscopic biopsy.

1. Dondalski M, White EM, Ghahremani GG, Patel SK. Carcinoma arising in urinary bladder diverticula: Imaging findings in six patients. AJR 1993;161(4):817-820.

2. Rincon VJM, Mdel PRC, Nivia MB, et al. Intradiverticular bladder tumor: CT assessment. Arch Esp Urol 2002;55(10):1235-1240.

3. Baniel J, Vishna T. Primary transitional cell carcinoma in vesical diverticula. Urology 1997;50(5):697-699. [http://dx.doi.org/10.1016/S0090-4295(97)00319-1]

4. Cheng CW, Tat MNG, Cheung HY, et al. Case report: Carcinosarcoma of the bladder diverticulum and a review of the literature. Int J Urology 2004;11(12):1136. [http://dx.doi.org/10.1111/ j.1442-2042.2004.00956.x]

5. Matta EJ, Kenney AK, Barré GM, Vanlangendonck Jr RM. Intradiverticular bladder carcinoma Radiographics 2005;25:1397-1403. [http://dx.doi.org/10.1148/rg.255045205] 